\title{
Depression and Anxiety One Month After Stroke
}

Asian/Pacific Island Nursing Journal

Volume 1(3): 82-90

(C)Author(s) 2016

http://digitalscholarship.unlv.edu/apin/

\author{
Cha-Nam Shin ${ }^{a}$, Mo-Kyung Sin ${ }^{b}$, Eunice Leec, Jongwon Leed, Kyungeh An ${ }^{\mathrm{e}}$, and Jeongha \\ $\operatorname{Sim}^{\mathrm{f}}$
}

\begin{abstract}
Depression and anxiety after stroke negatively affect patient outcomes; however, health care professionals may overlook poststroke depression and anxiety while they focus on the physical disabilities of patients soon after a stroke. The purpose of this study was to investigate the prevalence and predictors of depression, anxiety, or both concurrently at one month after stroke. We conducted a cross-sectional, descriptive study in a sample of 231 hospitalized patients with ischemic stroke in Korea. Data were collected by interviews using a series of structured questionnaires in addition to clinical data retrieved from patients' medical records. More than $70 \%$ were identified as depressed, $45.9 \%$ experienced anxiety, and $43.7 \%$ had concurrent depression and anxiety. Using a multiple logistic regression analysis, we identified anxiety as a predictor of depression; depression as a predictor of anxiety; and female sex, headaches, and swallowing difficulty as predictors of the comorbidity of depression and anxiety. Periodical screenings for poststroke depression and anxiety from an early stage in a hospital to years after stroke in a community are recommended to provide better chances for early identification of patients at risk because depression and anxiety may manifest at any stage of recovery. Special attention should be given to individuals with culturebound somatic symptoms in addition to female patients and those who have difficulty swallowing among Korean stroke patients.
\end{abstract}

Keywords: poststroke, depression, anxiety, predictors, Korean

Depression and anxiety are the most common mood disorders among stroke survivors with a prevalence of $13.7-31.1 \%$ for depression (American Stroke Association, 2014; Ayerbe, Ayis, Wolfe, \& Rudd, 2013; Broomfield, Quinn, Abdul-Rahim, Walters, \& Evans, 2014; Chriki, Bullain, \& Stern, 2006; Pedroso, de Souza, Brunont, \& Teixeira, 2015; Schöttke \& Giabbiconi, 2015) and 20.0-38.3\% for anxiety (American Stroke Association, 2014; Ayerbe, Ayis, Crichton, Wolfe, \& Rudd, 2014; Schöttke \& Giabbiconi, 2015). Depression or anxiety after stroke not only leads to poor involvement in rehabilitation and delays functional recovery, but results in limited social activity, poor quality of life, and increased disability (De Ryck et al., 2014; Skolarus, Burke, Brown, \& Freedman, 2014). Moreover, $12.3-73.2 \%$ of stroke survivors suffer from concurrent depression and anxiety (Ayerbe et al., 2014; Barker-Collo, 2007; Bergersen, Frøslie, Stibrant Sunnerhagen, \& Schanke, 2010), which further exacerbates recovery from stroke more than depression or anxiety alone after stroke (Ayerbe et al., 2014; Bergersen et al., 2010).
Studies have investigated contributing factors to the development of poststroke depression, but little is known about poststroke anxiety. Further, concurrent depression and anxiety have received limited attention. Therefore, the purposes of this study were to (1) investigate the prevalence of depression, anxiety, and concurrent depression and anxiety; (2) examine differences in depression, anxiety, and concurrent depression and anxiety by patient sociodemographic (e.g., age, sex) and clinical (e.g., impaired mobility) characteristics; and (3) determine predictors of having

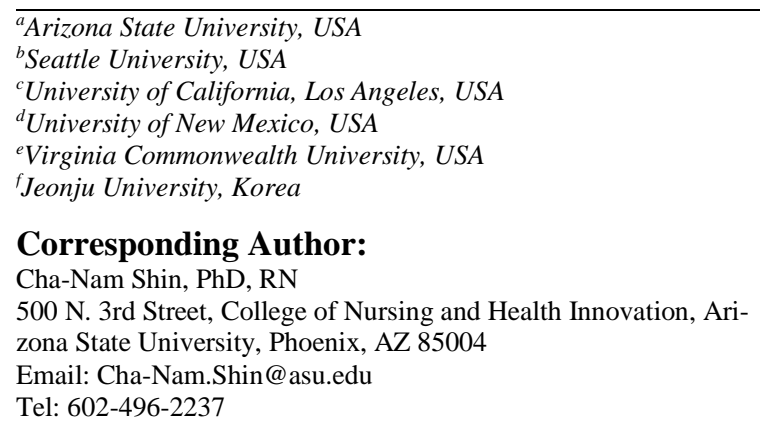

Corresponding Author:

Cha-Nam Shin, PhD, RN

500 N. 3rd Street, College of Nursing and Health Innovation, Arizona State University, Phoenix, AZ 85004

Email: Cha-Nam.Shin@asu.edu

Tel: 602-496-2237 
depression, anxiety, and concurrent depression and anxiety among ischemic stroke patients in Korea.

Mood disorders after stroke can occur at any stage of recovery (Ayerbe, Ayis, Rudd, Heuschmann, \& Wolfe, 2011; Chriki et al., 2006; Cichon, Bijak, Miller, Niwald, \& Saluk, 2015) and are often chronic and recurrent (Ayerbe et al., 2013; Choi-Kwon et al., 2012). The prevalence of poststroke depression (13.7$31.1 \%$ ) is 4 times higher than the likelihood of having depression in the general population without comorbid physical disease: $3.2 \%$ in individuals across 60 countries (Moussavi et al., 2007) and 6.7\% in U.S. adults (National Institute of Mental Health, 2016a). Poststroke depression increases the risk of recurrent stroke by 1.49 times (Yuan et al., 2012) and mortality by 1.22-1.52 times compared to nondepressed stroke patients (Bartoli et al., 2013). The prevalence of poststroke anxiety (20-38.3\%) is 6-8 times higher than the prevalence of anxiety disorder in the general population: 2.6-6.2\% across countries (Somers, Goldner, Waraich, \& Hsu, 2006) and 3.1\% in U.S. adults (National Institute of Mental Health, 2016b). The prevalence of concurrent depression and anxiety (12.3$73.2 \%$ ) is significantly higher in stroke survivors than in the general population (12.3-73.2\% vs. 5.6-51.2\%; Hirschfeld, 2001; Stordal, Bjelland, Dahl, Mykletun, $\&$ the Nord-Trøndelag Health Study, 2003; Winkler, Horáček, Weissová, Šustr, \& Brunovský, 2015).

When physical recovery is the main focus of treatment, the occurrence of depression and anxiety can be overlooked in the early stage of stroke recovery and thereafter (Chriki et al., 2006; De Ryck et al., 2014). Consequently, depression and anxiety are often poorly diagnosed and inadequately treated (Burton \& Tyson, 2015; De Ryck et al., 2014). Recognizing these symptoms are difficult because they often overlap with stroke-related impairments (Barker-Collo, 2007; Burton \& Tyson, 2015).

In Korea, cultural factors can complicate the ability of health care professionals to make an accurate diagnosis of depression and anxiety after stroke. Due to cultural values of modesty and harmony, Koreans tend to suppress their emotions and consider mood disorders as being out of balance and inner weaknesses (Bernstein, 2007; Pang, 1998). Therefore, symptoms of mood disorders in Koreans are often expressed by vague and unexplainable somatic symptoms such as body aches or headaches (Bernstein, 2007; Kramer, Kwong, Lee, \& Chung, 2002), which may make an early diagnosis after stroke challenging.

\section{Method}

\section{Design and Sample}

We conducted a cross-sectional, descriptive study in a sample of 231 patients hospitalized with neurologic symptoms of stroke and of ischemic stroke. The latter was determined as the primary diagnosis by brain-computed tomography or magnetic resonance imaging. The hospital was equipped to care for stroke patients and was located in a city in southwestern Korea. We obtained institutional review board approval from the study hospital and enrolled patients who were evaluated and referred by neurologists at the stroke clinic or neurology units of the hospital (see Figure 1).

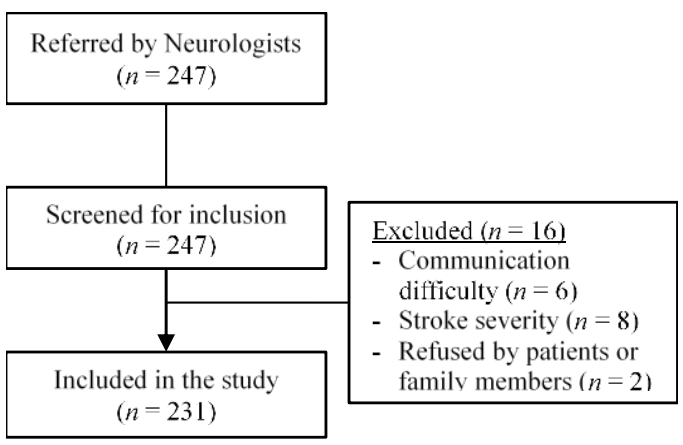

Figure 1. Recruitment Flow Diagram

Patients were eligible to participate if they were at 1 month after stroke onset and able to answer questions reliably without severe cognitive impairment or communication difficulties. The presence of severe cognitive or communication problems was screened using the clinical evaluation by the neurologists. We chose one month after stroke to assess depression and anxiety as soon after a stroke as possible to avoid screening in the acute stage (e.g., within 2-3 weeks after stroke). During the first 2-3 weeks, acute neurologic progression influences the patients' physical and cognitive conditions, which can lead to a high mortality rate or severe cognitive and communication problems that may result in a patient's inability to communicate and answer questions reliably (Aström, 1996; Choi-Kwon et al., 2012; Das et al., 2012).

After informed consent was obtained from each patient, two research assistants who had formal training sessions, including the interview protocol, conducted interviews with individual patients using structured questionnaires. The questionnaires consisted of a variety of items on patients' characteristics related to stroke. Patient responses were confirmed by a spouse or adult child who provided ancillary information and patients' medical records.

\section{Measures}

Data were collected on patients' sociodemographic characteristics, including age, sex, marital sta- 
tus, educational level, monthly family income, and living condition. Patients' clinical data such as impaired mobility, cognition, speech, and swallowing were retrieved from their medical records, which were initially determined by the neurologists' clinical evaluation at the study hospital. In addition, we asked about the most common symptoms stroke patients might have experienced, including the presence of headaches Patients were asked whether they were diagnosed with any chronic conditions (e.g., depression, anxiety, and impaired cognition or mobility) by health care professionals or taking medications for those conditions prior to the stroke.

We assessed depression and anxiety using the Hospital Anxiety and Depression Scale (HADS; Zigmond \& Snaith, 1983), which was available in Korean (Oh, Min, \& Park, 1999). HADS consists of two subscales (HADS-depression and HADS-anxiety) with 7 items each. Each item uses a 4-point Likert scale (from $0=$ not at all to $3=$ very often). Each subscale scores range 0-21, with higher scores indicating a greater likelihood of depression and anxiety. We used a score of 8 or above on the HADS-depression and HADSanxiety subscales as criteria for depression and anxiety, as recommended by the scale developers and consistent with previous research (Broomfield et al., 2014; Zigmond \& Snaith, 1983). HADS has shown acceptable reliability with Cronbach's alpha of 0.67 to 0.90 for HADS-depression and 0.68 to 0.93 for HADS-anxiety (Aben, Verhey, Lousberg, Lodder, \& Honig, 2002; Bjelland, Dahl, Haug, \& Neckelmann, 2002; Sagen et al., 2009). Likewise, the Korean version showed acceptable reliability with Cronbach's alpha of 0.86 for depression and 0.89 for anxiety in previous research (Oh et al., 1999) and our sample (Cronbach's alpha = 0.76 for depression and 0.88 for anxiety).

\section{Analytic Strategy}

Descriptive statistics were used to summarize sample characteristics. Chi-square tests were conducted to examine differences in the presence of depression, anxiety, and concurrent depression and anxiety after stroke by the patients' sociodemographic and clinical characteristics. To identify predictors of depression, anxiety, and concurrent depression and anxiety after stroke, we initially conducted a series of univariate logistic analyses for each independent variable. We then selected variables with $p<.10$ in bivariate logistic analyses and included them in the multivariate logistic regressions. SPSS Version 22 was used for data analysis, and an alpha of .05 was used to determine statistical significance.

\section{Results}

\section{Sample Characteristics}

The sample consisted mainly of men $(n=140$, $60.6 \%)$, with a mean age of $68.4(S D=10.6$, ranged $38-90$ years), who lived with a spouse $(n=160$, $69.3 \%$ ), had middle school or lower level of education $(57.8 \%)$, and $62.7 \%$ had a monthly family income of less than 1,000,000 Korean Won (about $\$ 920$ at the time of the study), which was considered to be low income. Impaired mobility was the most common symptom experienced by patients $(n=128,55.7 \%)$, followed by speech difficulties ( $n=89,39.4 \%)$, impaired cognition $(n=59,26 \%)$, headaches $(n=43,19.1 \%)$, and swallowing difficulty $(n=27,11.9 \%)$. None of the patients reported a history of impairment in mobility and cognition or taking medications for those conditions prior to the stroke, which was confirmed by their spouse or adult child.

The mean depression score was $10.11(S D=$ 4.24), and two-thirds (71.4\%) were identified as depressed. About half $(45.9 \%)$ had anxiety, and the mean anxiety score was $7.45(S D=4.95)$. Both depression and anxiety were identified using the recommended cut points: $\geq 8$ on the HADS- depression and anxiety subscales (Broomfield et al., 2014; Zigmond \& Snaith, 1983). While $26.4 \%(n=61)$ had neither depression nor anxiety, $43.7 \%(n=101)$ had both depression and anxiety, $27.7 \%(n=64)$ had depression only, and $2.2 \%$ $(n=5)$ had anxiety only. None of the patients reported a history of depression and anxiety or taking medications for those conditions prior to the stroke. Patients' responses were confirmed by their spouse or adult child. The correlation between depression and anxiety was $r=0.70(p<.001)$.

\section{Differences in Depression, Anxiety, and Concurrent Depression and Anxiety}

As presented in Tables 1-3, patients who were women; did not live with a spouse; experienced impairments in mobility, speech, cognition, or swallowing; and had anxiety were more likely to be depressed than men; lived with a spouse; did not experience impairments in mobility, speech, cognition, or swallowing; and did not have anxiety. Patients who were women; experienced impairments in speech, cognition, or swallowing; and had headaches and depression were more likely to demonstrate anxiety than did their counterparts. Patients who were women; experienced impairments in mobility, speech, cognition, or swallowing; and had headaches were more likely to have depression and anxiety concurrently compared to those who were men; did not experience impairments in mobility, speech, cognition, or swallowing; and did not have headaches. 
Table 1. Differences in Depression by Sample Characteristics

\begin{tabular}{|c|c|c|c|c|c|c|}
\hline Variable & Category & $\begin{array}{c}\text { Present }^{1} \\
n(\%)\end{array}$ & $\begin{array}{c}\text { Absent }^{2} \\
n(\%)\end{array}$ & $\begin{array}{l}\text { Total }^{3} \\
n(\%) \\
\end{array}$ & $\chi^{2}$ & $p$ \\
\hline \multicolumn{7}{|c|}{ Sociodemographic Characteristics } \\
\hline \multirow[t]{2}{*}{ Sex } & Male & $89(53.9)$ & $51(77.3)$ & $140(60.6)$ & 10.75 & .001 \\
\hline & Female & $76(46.1)$ & $15(22.7)$ & $91(39.4)$ & & \\
\hline \multirow[t]{2}{*}{ Age } & $\geq 61$ & $131(79.9)$ & $46(69.7)$ & $177(77.0)$ & 2.75 & .097 \\
\hline & $\leq 60$ & $33(20.1)$ & $20(30.3)$ & $53(23.0)$ & & \\
\hline \multirow[t]{2}{*}{ Living with spouse } & $\bar{Y}$ Yes & $105(63.6)$ & $55(83.3)$ & $160(69.3)$ & 8.59 & .003 \\
\hline & No & $60(36.4)$ & $11(16.7)$ & $71(30.7)$ & & \\
\hline \multirow[t]{2}{*}{ Educational status } & $\leq$ Middle school & $97(58.8)$ & $36(55.4)$ & $133(57.8)$ & .22 & .638 \\
\hline & $\geq$ High school & $68(41.2)$ & $29(44.6)$ & $97(42.2)$ & & \\
\hline \multirow[t]{2}{*}{ Income $^{3}$} & $<1,000,000 \mathrm{KRW}$ & $106(65.8)$ & $35(54.7)$ & $141(62.7)$ & 2.43 & .119 \\
\hline & $\geq 1,000,000 \mathrm{KRW}$ & $55(34.2)$ & $29(45.3)$ & $84(37.3)$ & & \\
\hline \multicolumn{7}{|c|}{ Clinical Characteristics } \\
\hline \multirow[t]{2}{*}{ Impaired mobility } & Yes & $102(61.8)$ & $26(40.0)$ & $128(55.7)$ & 8.99 & .003 \\
\hline & No & $63(38.2)$ & $39(60.0)$ & $102(44.3)$ & & \\
\hline \multirow{2}{*}{ Speech difficulty } & Yes & $74(45.7)$ & $15(23.4)$ & $89(39.4)$ & 9.51 & .002 \\
\hline & No & $88(54.3)$ & $49(76.6)$ & $137(60.6)$ & & \\
\hline \multirow[t]{2}{*}{ Impaired cognition } & Yes & $53(32.5)$ & $6(9.4)$ & $59(26.0)$ & 12.79 & $<.001$ \\
\hline & No & $110(67.5)$ & $58(90.6)$ & $168(74.0)$ & & \\
\hline \multirow[t]{2}{*}{ Headaches } & Yes & $35(21.7)$ & $8(12.5)$ & $43(19.1)$ & 2.53 & .112 \\
\hline & No & $126(78.3)$ & $56(87.5)$ & $182(80.9)$ & & \\
\hline \multirow[t]{2}{*}{ Swallowing difficulty } & Yes & $27(16.7)$ & $0(0)$ & 27 (11.9) & 12.11 & .001 \\
\hline & No & $135(83.3)$ & $64(100)$ & $199(88.1)$ & & \\
\hline \multirow[t]{2}{*}{ Anxiety } & Yes & $101(61.2)$ & $5(7.6)$ & $106(45.9)$ & 54.62 & $<.001$ \\
\hline & No & $64(38.8)$ & $61(92.4)$ & $125(54.1)$ & & \\
\hline
\end{tabular}

Note. ${ }^{1}$ present (HADS-depression score 8-21); ${ }^{2}$ absent (HADS-depression score 0-7); ${ }^{3}$ Family monthly income in Korean Won

Table 2. Differences in Anxiety by Sample Characteristics

\begin{tabular}{|c|c|c|c|c|c|c|}
\hline Variable & Category & $\begin{array}{c}\text { Present }^{1} \\
n(\%)\end{array}$ & $\begin{array}{c}\text { Absent }^{2} \\
n(\%)\end{array}$ & $\begin{array}{l}\text { Total }^{3} \\
n(\%)\end{array}$ & $\chi^{2}$ & $p$ \\
\hline \multicolumn{7}{|c|}{ Sociodemographic Characteristics } \\
\hline \multirow[t]{2}{*}{ Sex } & Male & $55(51.9)$ & $85(68.0)$ & $140(60.6)$ & 6.24 & .013 \\
\hline & Female & $51(48.1)$ & $40(32.0)$ & $91(39.4)$ & & \\
\hline \multirow[t]{2}{*}{ Age } & $\geq 61$ & $84(80.0)$ & $93(74.4)$ & $177(77.0)$ & 1.01 & .315 \\
\hline & $\leq 60$ & $21(20.0)$ & $32(25.6)$ & $53(23.0)$ & & \\
\hline \multirow[t]{2}{*}{ Living with spouse } & Yes & $68(64.2)$ & $92(73.6)$ & $160(69.3)$ & 2.41 & .121 \\
\hline & No & $38(35.8)$ & $33(26.4)$ & $71(30.7)$ & & \\
\hline \multirow[t]{2}{*}{ Educational status } & $\leq$ Middle school & $62(58.5)$ & $71(57.3)$ & $133(57.8)$ & .04 & .850 \\
\hline & $\geq$ High school & $44(41.5)$ & $53(42.7)$ & $97(42.2)$ & & \\
\hline \multirow[t]{2}{*}{ Income $^{3}$} & $<1,000,000 \mathrm{KRW}$ & $68(66.0)$ & $73(59.8)$ & $141(62.7)$ & .91 & .339 \\
\hline & $\geq 1,000,000 \mathrm{KRW}$ & $35(34.0)$ & $49(40.2)$ & $84(37.3)$ & & \\
\hline \multicolumn{7}{|c|}{ Clinical Characteristics } \\
\hline \multirow{2}{*}{ Impaired mobility } & Yes & $66(62.3)$ & $62(50.0)$ & $128(55.7)$ & 3.48 & .062 \\
\hline & No & $40(37.7)$ & $62(50.0)$ & $102(44.3)$ & & \\
\hline \multirow[t]{2}{*}{ Speech difficulty } & Yes & $51(49.5)$ & $38(30.9)$ & $89(39.4)$ & 8.14 & .004 \\
\hline & No & $52(50.5)$ & $85(69.1)$ & $137(60.6)$ & & \\
\hline \multirow[t]{2}{*}{ Impaired cognition } & Yes & $36(34.6)$ & $23(18.7)$ & $59(26.0)$ & 7.42 & .006 \\
\hline & No & $68(65.4)$ & $100(81.3)$ & $168(74.0)$ & & \\
\hline \multirow[t]{2}{*}{ Headaches } & Yes & $27(26.2)$ & $16(13.1)$ & $43(19.1)$ & 6.20 & .013 \\
\hline & No & $76(73.8)$ & $106(86.9)$ & $182(80.9)$ & & \\
\hline \multirow[t]{2}{*}{ Swallowing difficulty } & Yes & $21(20.4)$ & $6(4.9)$ & $27(11.9)$ & 12.82 & $<.001$ \\
\hline & No & $82(79.6)$ & $117(95.1)$ & $199(88.1)$ & & \\
\hline \multirow[t]{2}{*}{ Depression } & Yes & $101(95.3)$ & $64(51.2)$ & $165(71.4)$ & 54.62 & $<.001$ \\
\hline & No & $5(4.7)$ & $61(48.8)$ & $66(28.6)$ & & \\
\hline
\end{tabular}


Table 3. Differences in Concurrent Depression and Anxiety by Sample Characteristics

\begin{tabular}{|c|c|c|c|c|c|c|}
\hline Variable & Category & $\begin{array}{c}\text { Present }^{1} \\
n(\%) \\
\end{array}$ & $\begin{array}{c}\text { Absent }^{2} \\
n(\%)\end{array}$ & $\begin{array}{l}\text { Total }^{3} \\
n(\%) \\
\end{array}$ & $\chi^{2}$ & $p$ \\
\hline \multicolumn{7}{|c|}{ Sociodemographic Characteristics } \\
\hline \multirow{2}{*}{ Sex } & Male & $51(50.5)$ & $89(68.5)$ & $140(60.6)$ & 7.69 & .006 \\
\hline & Female & $50(49.5)$ & $41(31.5)$ & $91(39.4)$ & & \\
\hline \multirow[t]{2}{*}{ Age } & $\geq 61$ & $81(81.0)$ & $96(73.8)$ & $177(77.0)$ & 1.62 & .202 \\
\hline & $\leq 60$ & $19(19.0)$ & $34(26.2)$ & $53(23.0)$ & & \\
\hline \multirow[t]{2}{*}{ Living with spouse } & Yes & $64(63.4)$ & $96(73.8)$ & $160(69.3)$ & 2.93 & .087 \\
\hline & No & $37(36.6)$ & $34(26.2)$ & $71(30.7)$ & & \\
\hline \multirow[t]{2}{*}{ Educational status } & $\leq$ Middle school & $58(57.4)$ & $75(58.1)$ & $133(57.8)$ & .01 & .913 \\
\hline & $\geq$ High school & $43(42.6)$ & $54(41.9)$ & $97(42.2)$ & & \\
\hline \multirow[t]{2}{*}{ Income $^{3}$} & $<1,000,000 \mathrm{KRW}$ & $65(66.3)$ & $76(59.8)$ & $141(62.7)$ & .99 & .319 \\
\hline & $\geq 1,000,000 \mathrm{KRW}$ & $33(33.7)$ & $51(40.2)$ & $84(37.3)$ & & \\
\hline \multicolumn{7}{|c|}{ Clinical Characteristics } \\
\hline \multirow[t]{2}{*}{ Impaired mobility } & Yes & $64(63.4)$ & $64(49.6)$ & $128(55.7)$ & 4.34 & .037 \\
\hline & No & $37(36.6)$ & $65(50.4)$ & $102(44.3)$ & & \\
\hline \multirow[t]{2}{*}{ Speech difficulty } & Yes & $49(50.0)$ & $40(31.3)$ & $89(39.4)$ & 8.17 & .004 \\
\hline & No & $49(50.0)$ & $88(68.8)$ & $137(60.6)$ & & \\
\hline \multirow[t]{2}{*}{ Impaired cognition } & Yes & $35(35.4)$ & $24(18.8)$ & $59(26.0)$ & 8.00 & .005 \\
\hline & No & $64(64.6)$ & $104(81.3)$ & $168(74.0)$ & & \\
\hline \multirow[t]{2}{*}{ Headaches } & Yes & $26(26.5)$ & $17(13.4)$ & $43(19.1)$ & 6.18 & .013 \\
\hline & No & $72(73.5)$ & $110(86.6)$ & $182(80.9)$ & & \\
\hline \multirow[t]{2}{*}{ Swallowing difficulty } & Yes & $21(21.4)$ & $6(4.7)$ & $27(11.9)$ & 14.79 & $<.001$ \\
\hline & No & $77(78.6)$ & $122(95.3)$ & $199(88.1)$ & & \\
\hline
\end{tabular}

Note. ${ }^{1}$ present (HADS-depression score 8-21 and HADS-anxiety score 8-21); ${ }^{2}$ absent (HADS- depression scores 0-7 and HADSanxiety score $0-7) ;{ }^{3}$ Family monthly income in Korean Won

\section{Predictors of Depression, Anxiety, and Concurrent Depression and Anxiety}

In the multivariate logistic regression models, we identified anxiety as a predictor of depression, whereas depression as a predictor of anxiety. We also identified that female sex, headaches, and swallowing difficulty were predictors of the concurrence of depression and anxiety (see Table 4).

Table 4. Predictors of Depression, Anxiety, or Concurrent Depression and Anxiety

\begin{tabular}{|c|c|c|c|c|}
\hline Variable & Predictor (reference group) & $B$ & $O R(95 \% \mathrm{CI})$ & $p$ \\
\hline \multirow[t]{8}{*}{ Depression } & Sex (male) & .52 & $1.69(.69-4.11)$ & .250 \\
\hline & Age $(\leq 60)$ & .26 & $1.30(.56-3.05)$ & .545 \\
\hline & Without spouse (with spouse) & -.35 & $.71(.20-2.45)$ & .585 \\
\hline & Living alone (with family) & -.49 & $.61(.17-2.22)$ & .453 \\
\hline & Impaired mobility ${ }^{\mathrm{a}}$ & .41 & $1.50(.68-3.30)$ & .315 \\
\hline & Speech difficulty ${ }^{\mathrm{a}}$ & .65 & $1.91(.86-4.26)$ & .115 \\
\hline & Impaired cognition ${ }^{\mathrm{a}}$ & .80 & $2.22(.75-6.56)$ & .147 \\
\hline & Anxiety ${ }^{\mathrm{a}}$ & 2.72 & $15.15(5.61-40.88)$ & $<.001$ \\
\hline \multirow[t]{7}{*}{ Anxiety } & Sex (male) & .37 & $1.45(.76-2.76)$ & .258 \\
\hline & Swallowing difficulty ${ }^{\mathrm{a}}$ & .97 & $2.64(.92-7.60)$ & .071 \\
\hline & Impaired mobility & -.08 & $.92(.46-1.85)$ & .821 \\
\hline & Speech difficulty ${ }^{\mathrm{a}}$ & .23 & $1.26(.64-2.46)$ & .500 \\
\hline & Impaired cognition ${ }^{\mathrm{a}}$ & .30 & $1.35(.63-2.89)$ & .441 \\
\hline & Headaches $^{\mathrm{a}}$ & .73 & $2.08(.93-4.66)$ & .074 \\
\hline & Depression $^{\mathrm{a}}$ & 2.58 & $13.16(4.85-35.67)$ & $<.001$ \\
\hline \multirow[t]{7}{*}{ Concurrent } & Sex $($ male $)$ & .90 & $2.45(1.25-4.81)$ & .009 \\
\hline & Without spouse (with spouse) & .19 & $1.21(.59-2.46)$ & .604 \\
\hline & Swallowing difficulty ${ }^{\mathrm{a}}$ & 1.67 & $5.30(1.87-15.01)$ & .002 \\
\hline & Impaired mobility ${ }^{\mathrm{a}}$ & .16 & $1.17(.62-2.23)$ & .627 \\
\hline & Speech difficulty ${ }^{a}$ & .30 & $1.35(.72-2.52)$ & .354 \\
\hline & Impaired cognition ${ }^{\mathrm{a}}$ & .47 & $1.60(.76-3.35)$ & .214 \\
\hline & Headaches $^{\mathrm{a}}$ & .83 & $2.29(1.10-4.80)$ & .028 \\
\hline
\end{tabular}

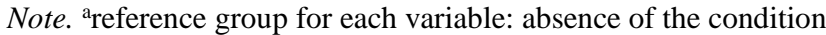




\section{Discussion}

Several important findings resulted from our study. The prevalence of poststroke depression or anxiety in this study was higher than the prevalence in previous studies: $71.4 \%$ versus $13.7-31.1 \%$ for depression and $45.9 \%$ versus $20.0-38.3 \%$ for anxiety (Ayerbe et al., 2013, 2014; Broomfield et al., 2014; Burton \& Tyson, 2015; Schöttke \& Giabbiconi, 2015). The prevalence of depression in our study was far greater than in other Korean stroke patients: 13.7-29.4 (Choi-Kwon et al., 2012; Jeong et al., 2014; Park, Im, Oh, Lee, \& Pae, 2015) or in the general Korean population without the comorbidity of physical diseases (3.5-4.0\%; Cho \& Lee, 2005; Oh et al., 2013). The prevalence of concurrent depression and anxiety after stroke $(43.7 \%)$ was much higher in our study than at 3 months after stroke (12.3\%; Barker-Collo, 2007).

Increased prevalence over the course of recovery such as $12.3 \%$ at 3 months (Barker-Collo, 2007), $17.3 \%$ at $2-5$ years (Bergersen et al., 2010), and $56.9-73.2 \%$ at $2-10$ years after stroke (Ayerbe et al., 2014) indicates that the negative health outcomes due to depression and anxiety after stroke could be continued across stages of recovery and even more significant in later stages. These findings suggest that screenings for both depression and anxiety should be included in a routine checkup from an early stage in a hospital (i.e., at 1 month) to years after stroke in a community. This will provide better opportunities for early identification of patients at risk because depression and anxiety may manifest at any stage of recovery (Chriki et al., 2006; Gillham \& Clark, 2011).

Consistent with reports of research in the general population (Stordal et al., 2003) and stroke patients (Ayerbe et al., 2014; De Ryck et al., 2014), patients who were women and experienced impairments in physical or cognitive functions were more likely to exhibit depression, anxiety, or concurrent depression and anxiety in this study. The high correlation $(r=$ $0.70)$ between depression and anxiety in our study was similar to the correlations $(0.59-0.63)$ reported in other studies (Barker-Collo, 2007; Bergersen et al., 2010). In line with prior studies, poststroke anxiety was a significant predictor of poststroke depression (Ayerbe et al., 2013; Morrison, Pollard, Johnston, \& MacWalter, 2005; Schöttke \& Giabbiconi, 2015), and poststroke depression was a significant predictor of poststroke anxiety (Kroeders, Benhardt, \& Cumming, 2013). Our findings and reports in previous studies support the recursive interconnections linking the brain regions involved in anxiety and depression (Zbozinek et al., 2012).

We found that a headache was among the predictors of concurrent depression and anxiety. This could be explained by the fact that a headache is a somatically expressed mood disorders, which is culturally acceptable among Koreans (Bernstein, 2007; Kramer et al., 2002; Pang, 1998). Also, a headache is among the risk factors or warning signs of stroke (Katsarava \& Weimar, 2010). Therefore, it is possible that participants who have experienced a headache at the onset of stroke might have felt anxious about having another stroke by connecting a headache with a stroke when they had a persistent headache after stroke.

Our study has several strengths. First, we used the HADS, a valid and reliable instrument to measure depression and anxiety after stroke. Unlike other screening tools, HADS does not include items reflecting somatic symptoms of stroke such as fatigue and insomnia so that health care professionals are able to assess mood disorders independently from strokerelated somatic deficits (Barker-Collo, 2007; Berg, Lönnqvist, Palomäki, \& Kaste, 2009; Bjelland et al., 2002). Also, HADS is a simple, brief, standardized tool; therefore, it can be easily adopted in any health care setting (Gillham \& Clark, 2011). Second, we carefully considered the screening time (one month after stroke) to assess mood disorders as soon after a stroke as possible but without or less influence of acute neurologic progression on the patients' physical and cognitive conditions. Consequently, we could obtain reliable answers from patients. Third, our study is one of the few research that has investigated predictors of concurrent depression and anxiety in addition to predictors of poststroke depression or anxiety among ischemic stroke patients. Therefore, our study extends the growing body of scientific knowledge on poststroke depression and anxiety.

\section{Limitations}

Due to the nature of the study design (i.e., a cross-sectional, descriptive study), we could not capture the sequence of depression and anxiety onsets and their changes over the course of recovery. Also, the study findings may not be generalizable to stroke patients in other populations or settings because the data were collected at a single location in Korea. We did not include other factors associated with poststroke depression or anxiety such as stroke location or volume. Therefore, the findings should be interpreted with caution. Self-report of pre-existing conditions (e.g., depression, anxiety, and impairments in cognition or mobility) may be another potential limitation of our study. To enhance reliability, we confirmed patients' responses by their spouse and adult child who might readily identify or suspect those conditions as the closest family members. 


\section{Conclusion}

Future research with a longitudinal research design and adequate follow-ups (e.g., 6 months, 12 months, and 3 years) would provide a better understanding of the sequence of depression and anxiety onsets and changes over the course of recovery among stroke survivors. Further exploration of somatic symptoms (i.e., headaches) of depression and anxiety in the cultural context could lead to a deeper understanding of depression and anxiety in Korean stroke patients. Given that the reciprocal relationship between depression and anxiety (i.e., depression predicts anxiety, and vice versa) and the significant percentage (43.7\%) of patients experienced both mood disorders concurrently after their stroke, interventions should target both depression and anxiety, rather than targeting them separately. This may have a synergistic effect on both depression and anxiety and health outcomes among stroke survivors.

Particular attention should be paid to female patients and those who have difficulty swallowing and headaches, as these patients were at a higher risk for concurrent depression and anxiety. Additional attention to patients' expression of culture-bound somatic symptoms (e.g., headaches) will lead to an integrated and holistic assessment of individuals who cannot directly express symptoms of depression and anxiety after stroke. This approach may be critical because displays of negative emotions (e.g., depression) through somatic symptoms (e.g., a headache) are culturally and socially acceptable in Korean culture and most Asian cultures where group harmony is valued (Grover \& Ghosh, 2014; Kalibatseva \& Leong, 2011; Saint Arnault \& Kim, 2008). In contrast, direct display of emotional distress is favored in Western cultures (e.g., the United States) where independence and individualism are valued (Kalibatseva \& Leong, 2011; Saint Arnault \& Kim, 2008).

In conclusion, this study highlights a high prevalence of poststroke depression, anxiety, and concurrent depression and anxiety among ischemic stroke patients in Korea. Considering the negative impact of depression, anxiety, and concurrent depression and anxiety on patient outcomes, early and periodic assessments using a valid and reliable screening tool will promote the timely recognition of individuals who have these mood disorders. Such assessments may lead to appropriate treatments and ultimately improve patients' health outcomes, including reduction of disability and mortality after stroke.

\section{Source of Funding}

Dr. Jeongha Sim received a grant from the Basic Research Program through the National Research Foundation of Korea (NRF) funded by the Ministry of Education, Science and Technology (2011-0014173) in 2011.

\section{Conflict of Interest}

The authors declare no conflicts of interest.

\section{Acknowledgement}

The authors gratefully acknowledge the editorial assistance from Dr. Nancy Moore in the preparation of the manuscript.

\section{References}

Aben, I., Verhey, F., Lousberg, R., Lodder, J., \& Honig, A. (2002). Validity of the Beck Depression Inventory, Hospital Anxiety and Depression Scale, SCL-90, and Hamilton Depression Rating Scale as screening instruments for depression in stroke patients. Psychosomatics, $\quad$ 43, 386-393. doi:10.1176/appi.psy.43.5.386

American Stroke Association. (2014). Post-stroke mood disorders. Retrieved from http://www.strokeassociation.org/STROKEORG/LifeAfterStroke/RegainingIndependence/EmotionalBehavioralChallenges/Post-Stroke-Mood-isorders_UCM_467460_Article.jsp

Aström, M. (1996). Generalized anxiety disorder in stroke patients. A 3-year longitudinal study. Stroke, 27, 270-275. doi:10.1161/01.STR.27.2.270

Ayerbe, L., Ayis, S. A., Crichton, S., Wolfe, C. D., \& Rudd, A. G. (2014). Natural history, predictors and associated outcomes of anxiety up to 10 years after stroke: The South London Stroke Register. Age and Ageing, 43, 542-547. doi:10.1093/ageing/aft208

Ayerbe, L., Ayis, S., Rudd, A. G., Heuschmann, P. U., \& Wolfe, C. D. (2011). Natural history, predictors, and associations of depression 5 years after stroke. Stroke, 42, 1907-1911. doi:10.1161/STROKEAHA.110.605808

Ayerbe, L., Ayis, S., Wolfe, C. D., \& Rudd, A. G. (2013). Natural history, predictors and outcomes of depression after stroke: Systematic review and metaanalysis. British Journal of Psychiatry, 202, 14-21. doi:10.1192/bjp.bp.111.107664.

Barker-Collo, S. L. (2007). Depression and anxiety 3 months post stroke: Prevalence and correlates. Archives of Clinical Neuropsychology, 22, 519-531. doi:10.1016/j.acn.2007.03.002

Bartoli, F., Lillia, N., Lax, A., Crocamo, C., Mantero, V., Carrà, G., ... Clerici, M. (2013). Depression after stroke and risk of mortality: A systematic review and meta-analysis. Stroke Research and Treatment, 2013, 862978-862988. doi:10.1155/2013/862978 
Berg, A., Lönnqvist, J., Palomäki, H., \& Kaste, M. (2009). Assessment of depression after stroke: A comparison of different screening instruments. Stroke, 40, 523-529. doi:10.1161/STROKEAHA.108.527705

Bergersen, H., Frøslie, K. F., Stibrant Sunnerhagen, K., \& Schanke, A.-K. (2010). Anxiety, depression, and psychological well-being 2 to 5 years poststroke. Journal of Stroke and Cerebrovascular Diseases, 19, 364-369. doi:10.1016/j.jstrokecerebrovasdis.2009.06.005

Bernstein, K. S. (2007). Mental health issues among urban Korean American immigrants. Journal of Transcultural Nursing, 18, 175-180. doi:10.1177/1043659606298610

Bjelland, I., Dahl, A. A., Haug, T. T., \& Neckelmann, D. (2002). The validity of the Hospital Anxiety and Depression Scale: An updated literature review. Journal of Psychosomatic Research, 52, 69-77. doi:10.1016/S0022-3999(01)00296-3

Broomfield, N. M., Quinn, T. J., Abdul-Rahim, A. H., Walters, M. R., \& Evans, J. J. (2014). Depression and anxiety symptoms post-stroke/TIA: Prevalence and associations in cross-sectional data from a regional stroke registry. BMC Neurology, 14, 198. doi:10.1186/s12883-014-0198-8

Burton, L.-J., \& Tyson, S. (2015). Screening for mood disorders after stroke: A systematic review of psychometric properties and clinical utility. Psychological Medicine, 45, 29-49. doi:10.1017/S0033291714000336

Cho, M. J., \& Lee, J. Y. (2005). Epidemiology of depressive disorders in Korea. Psychiatry Investigation, 2, 22-27.

Choi-Kwon, S., Han, K., Choi, S., Suh, M., Kim, Y.-J., Song, H., ... Kim, J. S. (2012). Poststroke depression and emotional incontinence: Factors related to acute and subacute stages. Neurology, 78, 1130-1137. doi:10.1212/WNL.0b013e31824f8090

Chriki, L. S., Bullain, S. S., \& Stern, T. A. (2006). The recognition and management of psychological reactions to stroke: A case discussion. Primary Care Companion to the Journal of Clinical Psychiatry, 8, 234-240. doi:10.4088/PCC.v08n0407

Cichoń, N., Bijak, M., Miller, E., Niwald, M., \& Saluk, J. (2015). Poststroke depression as a factor adversely affecting the level of oxidative damage to plasma proteins during a brain stroke. Oxidative Medicine and Cellular Longevity, 2015, 408745. doi:10.1155/2015/408745

Das, S., Chandra Ghosh, K., Malhotra, M., Yadav, U., Sankar Kundu, S., \& Kumar Gangopadhyay, P. (2012). Short term mortality predictors in acute stroke. Annals of Neurosciences, 19, 61-67. doi:10.5214/ans.0972.7531.12190203

De Ryck, A., Brouns, R., Geurden, M., Elseviers, M., De Deyn, P. P., \& Engelborghs, S. (2014). Risk factors for poststroke depression: Identification of inconsistencies based on a systematic review. Journal of Geriatric Psychiatry and Neurology, 27, 147-158. doi:10.1177/0891988714527514

Gillham, S., \& Clark, L. (2011). Psychological care after stroke. Retrieved from https://www.nice.org.uk/media/de-

fault/sharedlearning/531_strokepsychologicalsupportfinal.pdf.

Grover, S., \& Ghosh, A. (2014). Somatic symptom and related disorders in Asians and Asian Americans. Asian Journal of Psychiatry, 7, 77-79. doi:10.1016/j.ajp.2013.11.014

Hirschfeld, R. M. A. (2001). The comorbidity of major depression and anxiety disorders: Recognition and management in primary care. Primary Care Companion to the Journal of Clinical Psychiatry, 3, 244-254. doi:10.4088/PCC.v03n0609

Jeong, Y. J., Kim, W. C., Kim, Y. S., Choi, K. W., Son, S. Y., \& Jeong, Y. G. (2014). The relationship between rehabilitation and changes in depression in stroke patients. Journal of Physical Therapy Science, 26, 1263-1266. doi:10.1589/jpts.26.1263

Kalibatseva, Z, \& Leong, F. T. L. (2011) Depression among Asian Americans: Review and recommendations. Depression Research and Treatment, 2011, 320902. doi:10.1155/2011/320902

Katsarava, Z., \& Weimar, C. (2010). Migraine and stroke. Journal of the Neurological Sciences, 299, 42-44. doi:10.1016/j.jns.2010.08.058

Kramer, E. J., Kwong, K., Lee, E., \& Chung, H. (2002). Cultural factors influencing the mental health of Asian Americans. Western Journal of Medicine, 176, 227-231.

Kroeders, R., Benhardt, J., \& Cumming, T. (2013). Physical inactivity, depression and anxiety in acute stroke. International Journal of Therapy and Rehabilitation, 20, 289-293. doi:10.12968/ijtr.2013.20.6.289

Morrison, V., Pollard, B., Johnston, M., \& MacWalter, R. (2005). Anxiety and depression 3 years following stroke: Demographic, clinical, and psychological predictors. Journal of Psychosomatic Research, 59, 209-213. doi:10.1016/j.jpsychores.2005.02.019

Moussavi, S., Chatterji, S., Verdes, E., Tandon, A., Patel, V., \& Ustun, B. (2007). Depression, chronic diseases, and decrements in health: Results from the World Health Surveys. Lancet, 370, 851-858. doi:10.1016/S0140-6736(07)61415-9

National Institute of Mental Health. (2016a). Major depression among adults. Retrieved from http://www.nimh.nih.gov/health/statistics/prevalence/major-depression-among-adults.shtml

National Institute of Mental Health. (2016b). Generalized anxiety disorder among adults. Retrieved from http://www.nimh.nih.gov/health/statistics/prevalence/generalized-anxiety-disorder-amongadults.shtml

Oh, D. H., Kim, S. A., Lee, H. Y., Seo, J. Y., Choi, B-Y., \& Nam J. H. (2013). Prevalence and correlates of depressive symptoms in Korean adults: Results of a 2009 Korean community health survey. Journal of Korean Medical Science, 28, 128-135. doi:10.3346/jkms.2013.28.1.128

Oh, S. M., Min, K. J., \& Park, D. B. (1999). A study on the standardization of the Hospital Anxiety and De- 
pression Scale for Koreans: A comparison of normal, depressed and anxious groups. Journal of Korean Neuropsychiatric Association, 38, 289-296.

Pang, K. Y. (1998). Symptoms of depression in elderly Korean immigrants: Narration and the healing process. Culture, Medicine and Psychiatry, 22, 93122. doi:10.1023/A:1005389321714

Park, G. Y., Im, S., Oh, C. H., Lee, S. J., \& Pae, C. U. (2015). The association between the severity of poststroke depression and clinical outcomes after first-onset stroke in Korean patients. General Hospital Psychiatry, $\quad 37, \quad 245-250$. doi:10.1016/j.genhosppsych.2015.02.009

Pedroso, V. S. P., de Souza, L. C., Brunont, A. R., \& Teixeira A. L. (2015). Post stroke depression: Clinics, etiopathogenesis and therapeutics. Archives of Clinical Psychiatry, 42, 18-24. doi:10.1590/010160830000000041

Sagen, U., Vik, T. G., Moum, T., Mørland, T., Finset, A., \& Dammen, T. (2009). Screening for anxiety and depression after stroke: Comparison of the Hospital Anxiety and Depression Scale and the Montgomery and Asberg Depression Rating Scale. Journal of Psychosomatic Research, 67, 325-332. doi:10.1016/j.jpsychores.2009.03.007

Saint Arnault, D., \& Kim, O. (2008). Is there an Asian idiom of distress? Somatic symptoms in female Japanese and Korean students. Archives of Psychiatric Nursing, 22, 27-38. doi:10.1016/j.apnu.2007.10.003

Schöttke, H., \& Giabbiconi, C. M. (2015). Post-stroke depression and post-stroke anxiety: Prevalence and predictors. International Psychogeriatrics, 27, 1805-1812. doi:10.1017/S1041610215000988

Skolarus, L. E., Burke, J. F., Brown, D. L., \& Freedman, V. A. (2014). Understanding stroke survivorship: Expanding the concept of poststroke disability.
Stroke, 45,
doi:10.1161/STROKEAHA.113.002874

Somers, J. M., Goldner, E. M., Waraich, P., \& Hsu, L. (2006). Prevalence and incidence studies of anxiety disorders: A systematic review of the literature. Canadian Journal of Psychiatry, 51, 100-113.

Stordal, E., Bjelland, I., Dahl, A. A., Mykletun, A., \& the Nord-Trøndelag Health Study (HUNT). (2003). Anxiety and depression in individuals with somatic health problems. Scandinavian Journal of Primary Health Care, 21, 136-141. doi:10.1080/02813430310002030

Winkler, P., Horáček, J., Weissová, A., Šustr, M., \& Brunovský, M. (2015). Physical comorbidities in depression co-occurring with anxiety: A cross sectional study in the Czech primary care system. International Journal of Environmental Research and Public Health, 12, 15728-15738. doi:10.3390/ijerph121215015

Yuan, H. W., Wang, C. X., Zhang, N., Bai, Y., Shi, Y. Z., Zhou, Y., ... Wang, Y. J. (2012). Poststroke depression and risk of recurrent stroke at 1 year in a Chinese cohort study. PLoS ONE, 7(10), e46906. doi:10.1371/journal.pone.0046906

Zbozinek, T. D., Rose, R. D., Wolitzky-Taylor, K. B., Sherbourne, C., Sullivan, G., Stein, M. B., ... Craske, M. G. (2012). Diagnostic overlap of generalized anxiety disorder and major depressive disorder in a primary care sample. Depression and Anxiety, 29, 1065-1071. doi:10.1002/da.22026

Zigmond, A. S., \& Snaith, R. P. (1983). The Hospital Anxiety and Depression Scale. Acta Psychiatrica Scandinavica, 67, 361- 370. doi:10.1111/j.16000447.1983.tb09716.x 